LA-UR-01-0091

Approved for public release: distribution is unlimited.

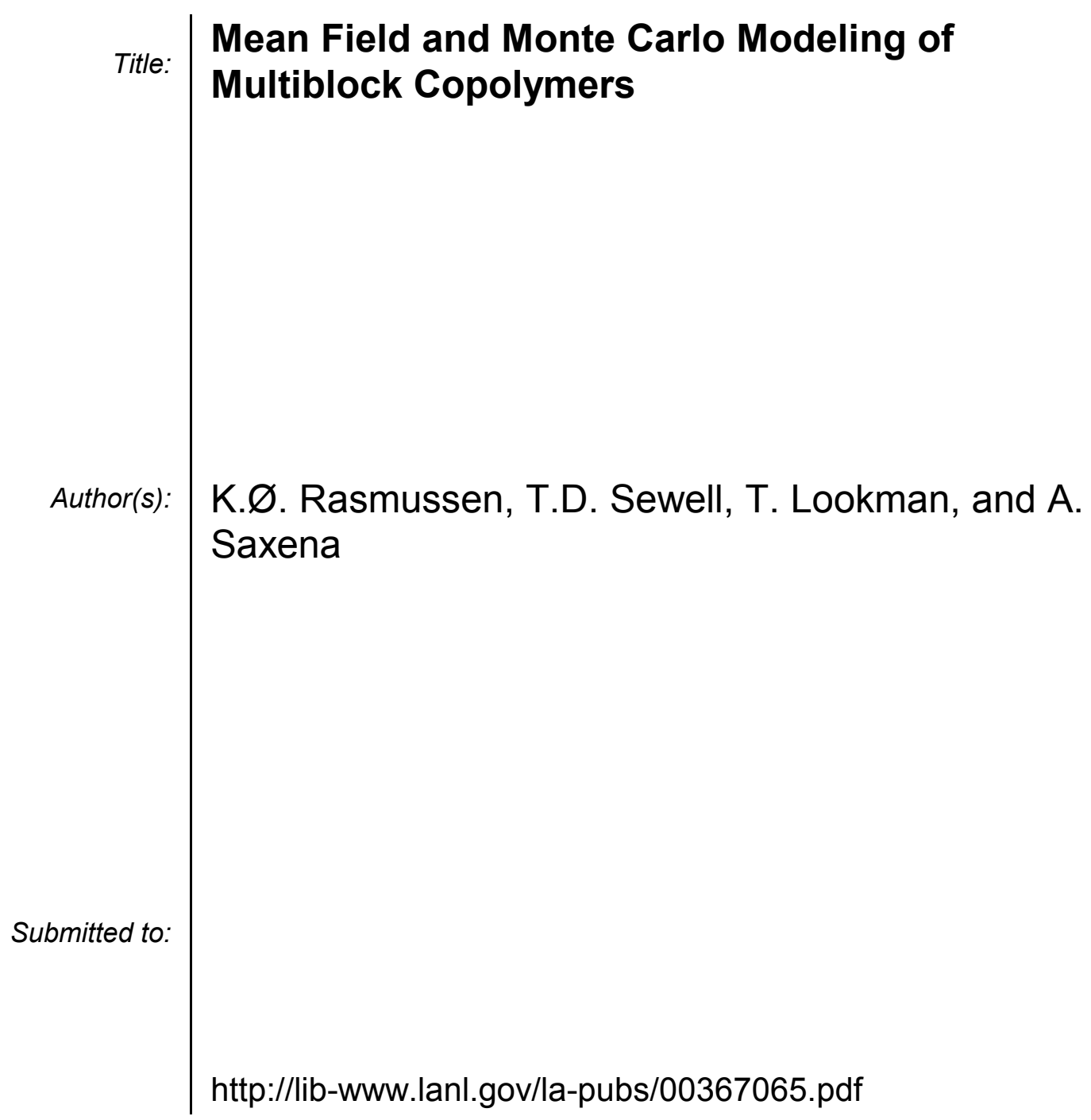

Los Alamos National Laboratory, an affirmative action/equal opportunity employer, is operated by the University of California for the U.S. Department of Energy under contract W-7405-ENG-36. By acceptance of this article, the publisher recognizes that the U.S. Government retains a nonexclusive, royaltyfree license to publish or reproduce the published form of this contribution, or to allow others to do so, for U.S. Government purposes. Los Alamos National Laboratory requests that the publisher identify this article as work performed under the auspices of the U.S. Department of Energy. Los Alamos National Laboratory strongly supports academic freedom and a researcher's right to publish; as an institution, however, the Laboratory does not endorse the viewpoint of a publication or guarantee its technical correctness. 


\title{
Mean Field and Monte Carlo Modeling of Multiblock Copolymers
}

\author{
K. Ø. Rasmussen, T. D. Sewell, T. Lookman, and A. Saxena \\ Theoretical Division, Los Alamos National Laboratory, Los Alamos, NM 87545
}

\begin{abstract}
We discuss and apply extensions needed to treat multiblock copolymers within the mean field theoretical framework for microphase separation in diblock copolymer melts, originally due to Leibler. The mean field calculations are complemented by lattice Monte Carlo realizations using the bond fluctuation model. We find that the microphase separation transition occurs at larger $\chi N$ as the number of blocks in increased beyond two (i.e., beyond diblock), and that the characteristic length scale of the emerging morphology decreases as the number of blocks increases. The latter prediction is in qualitative agreement with published experimental results due to Spontak and co-workers for model multiblock poly(styrene-isoprene) systems and recent results due to Hjelm and co-workers for a segmented poly(ester-urethane) relevant to Los Alamos interests. Additionally, the mean field predictions and bond fluctuation realizations yield consistent results.
\end{abstract}

\section{Introduction}

The elastic scattering of light, $\mathrm{x}$-rays, and neutrons due to thermal concentration fluctuations in homopolymer melts is one of the more interesting subjects in polymer physics because the fluctuations reflect microscopic properties of single chains such as the radius of gyration as well as thermodynamic interactions between monomers such as the Flory-Huggins interaction parameter $\chi$. In the case of blends, copolymers, etc., analysis of the fluctuations leads to information about, and improved understanding of, the microphase separation transition (MST) in multicomponent systems.

One of the most general and widely applied theories for the MST in diblock systems was developed by Leibler in the context of the random phase approximation. In the present report we describe extensions to and applications of the theory to treat multiblock systems $\left[A_{m} B_{n}\right]_{p}$, where $(m+n) p=N$ is the total chain length. Of particular interest are (1) the $\chi N$ dependence of the MST and (2) the variation of characteristic length scale for concentration fluctuations based on peak positions in the structure factor, both as functions of the block number $p$ and the ratio $\phi=m /(m+n)$. The mean field predictions for the structure factors are compared in selected cases to the results of lattice Monte Carlo realizations performed using the bond fluctuation algorithm with identical values of $N, \phi$, and $\chi$. This provides for an internal consistency check between the two approaches and sets the stage for more thorough studies of the effects of "aging" on chain level details of polymer morphology and other properties. 


\section{Mean Field Model}

In this section we apply the formalism initially developed by L. Leibler [1] and Kawasaki and co-workers [2] for diblock copolymers systems. The method starts from the microscopic Edwards Hamiltonian [3] for a two component system consisting of $n$ polymer chains composed of $f$ monomers of type $\mathrm{A}$ and $N-f$ monomers of type $\mathrm{B}$

$$
H=\frac{1}{2} \sum_{i=1}^{n} \int_{0}^{N} d \tau\left(\frac{d \mathbf{r}_{i}(\tau)}{d \tau}\right)^{2}+V\left\{\mathbf{r}_{\mathbf{i}}\right\}
$$

where the excluded volume interaction $V\left\{\mathbf{r}_{\mathbf{i}}\right\}$ is

$$
\begin{aligned}
V\left\{\mathbf{r}_{\mathbf{i}}\right\} & =\frac{\epsilon_{A A}}{2} \sum_{i, j}^{n} \int_{0}^{f} d \tau \int_{0}^{f} d \tau^{\prime} \delta\left(\mathbf{r}_{i}(\tau)-\mathbf{r}_{j}\left(\tau^{\prime}\right)\right) \\
& +\frac{\epsilon_{B B}}{2} \sum_{i, j}^{n} \int_{f}^{N} d \tau \int_{f}^{N} d \tau^{\prime} \delta\left(\mathbf{r}_{i}(\tau)-\mathbf{r}_{j}\left(\tau^{\prime}\right)\right) \\
& +\frac{\epsilon_{A B}}{2} \sum_{i, j}^{n} \int_{f}^{N} d \tau \int_{0}^{f} d \tau^{\prime} \delta\left(\mathbf{r}_{i}(\tau)-\mathbf{r}_{j}\left(\tau^{\prime}\right)\right) .
\end{aligned}
$$

The monomer-monomer interaction strengths $\epsilon_{A A}, \epsilon_{A B}$, and $\epsilon_{B B}$ are assumed positive. If we now introduce the monomer densities

$$
\rho_{A}=\sum_{i=1}^{n} \frac{1}{\rho_{0}} \int_{0}^{f} d \tau \delta\left(\mathbf{r}-\mathbf{r}_{i}(\tau)\right), \quad \text { and } \quad \rho_{B}=\sum_{i=1}^{n} \frac{1}{\rho_{0}} \int_{f}^{N} d \tau \delta\left(\mathbf{r}-\mathbf{r}_{i}(\tau)\right)
$$

assume incompressibility $\rho_{A}(\mathbf{r})+\rho_{B}(\mathbf{r})=\rho_{0}=$ const, and define the local deviation of the monomer density $\psi(\mathbf{r}) \equiv \rho_{A}(\mathbf{r})-\rho_{0} \frac{f}{N}=-\rho_{B}(\mathbf{r})+\rho_{0} \frac{N-f}{N}$ the interaction part of the microscopic Hamiltonian (1) can be rewritten as

$$
V\left\{\mathbf{r}_{i}\right\}=-\chi \int \psi^{2}(\mathbf{r}) d \mathbf{r}
$$

where $\chi=\epsilon_{A B}-\frac{1}{2}\left(\epsilon_{A A}+\epsilon_{B B}\right)$ is the Flory-Huggins parameter [4]. To account for the entropic effects we can continue in a coarse grained picture by writing down the partition function for the system

$$
\begin{aligned}
Z & =\int d\left\{\mathbf{r}_{i}\right\} \int d\{\psi\} \times\left[\prod_{r} \delta\left(\psi+\frac{f}{N}-\sum_{i=1}^{n} \int_{0}^{f} d \tau \delta\left(\mathbf{r}-\mathbf{r}_{i}(\tau)\right)\right)\right] \\
& \times\left[\prod_{r} \delta\left(\rho_{0}-\sum_{i=1}^{n} \int d \tau \delta\left(\mathbf{r}-\mathbf{r}_{i}(\tau)\right)\right)\right] \times \exp \left[-H_{0}\{\mathbf{r}\}+\chi \int \psi^{2} d \mathbf{r}\right]
\end{aligned}
$$

where the incompressibility has been taken into account explicitly by the first $\delta$-function. Using the approximation know as the Random Phase Approximation [4] introduced into soft condensed matter physics by de Gennes the spatial integration can be approximately carried out, resulting in the following free energy $F\{\psi\}=k T \log Z$

$$
F=\int d \mathbf{r} \int d \mathbf{r}^{\prime} \Gamma\left(\mathbf{r}, \mathbf{r}^{\prime}\right) \psi(\mathbf{r}) \psi\left(\mathbf{r}^{\prime}\right)-\chi \int \psi^{2}(\mathbf{r}) d \mathbf{r}+\mathcal{W}\{\psi\}
$$



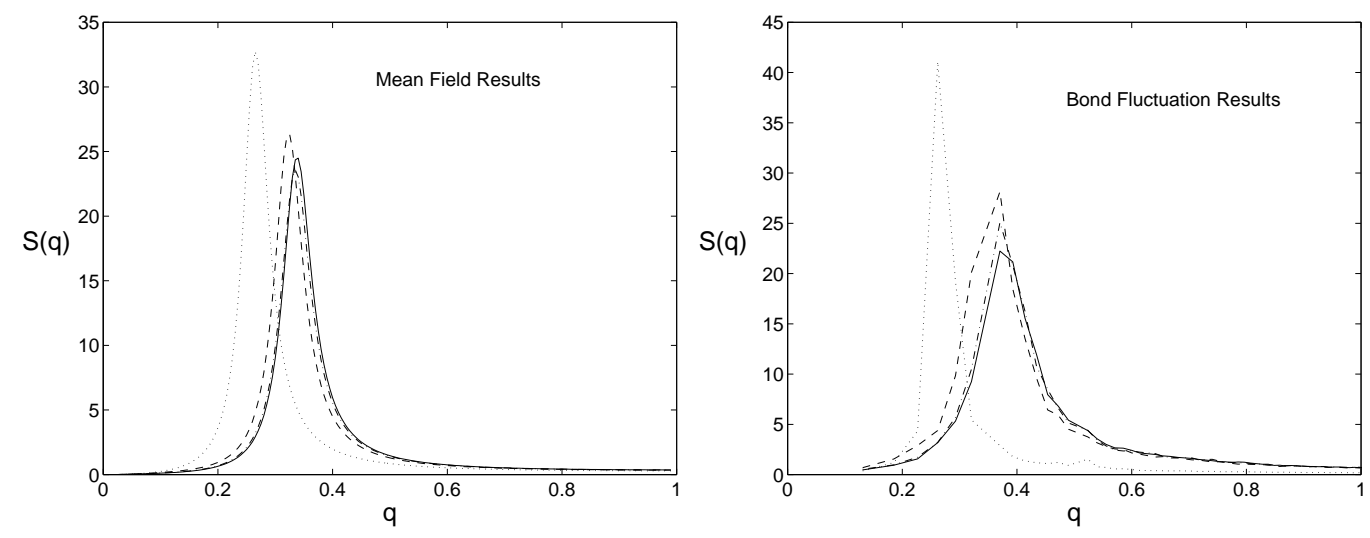

Figure 1: Mean field (left) and Bond fluctuation method (right) structure factors for multiblock copolymers $\left(A_{m} B_{n}\right)_{p}$. Dotted lines: $p=2$ (diblock), dashed lines: $p=3$, dash dotted lines: $p=5$, and solid lines: $p=6$.

where $\mathcal{W}$ denotes terms higher order in $\psi$. In this context we will not need to consider these terms in further detail. The coefficient of the squared term comes via the RPA out to be

$$
\Gamma(\mathbf{q})=S_{A A}^{-1}(\mathbf{q})-2 S_{A B}^{-1}(\mathbf{q})+S_{B B}^{-1}(\mathbf{q}),
$$

where $S_{A A}(\mathbf{q}), S_{A B}(\mathbf{q})$, and $S_{B B}(\mathbf{q})$ are the monomer-monomer structure factors which we will define mathematically below. It is important to note that these structure factors transform as matrix elements not as simple scalars

$$
\left\{S_{i j}^{-1}\right\}\left\{S_{i j}\right\}=\mathbf{I}
$$

where $\mathbf{I}$ is the identity matrix. The structure factor, $S(\mathbf{q})$ for the entire system can be read off Eq. (5) to be

$$
S(\mathbf{q})=\frac{1}{\Gamma(\mathbf{q})-\chi}
$$

Up to this point the theory was developed in the context of diblock copolymers. However, the formalism is valid for any structure, but the monomer-monomer structure factors has to be calculated for the particular architecture one has in mind. For a linear multiblock copolymer the monomer-monomer structure factors are given as

$$
S_{i j}(\mathbf{q})=\int_{0}^{N} d \tau \int_{0}^{N} d \tau^{\prime} \theta_{i}(\tau) \theta_{j}\left(\tau^{\prime}\right) \exp \left(-\frac{b^{2}}{6} q^{2}\left|\tau-\tau^{\prime}\right|\right)
$$

where $\theta_{i}(\tau)$ is 1 whenever the $\tau^{\prime}$ th monomer is of type $i$ (= A or B) and 0 otherwise.

Using this developed formalism we can immediately extract useful information on the phase behavior of multiblock copolymers. In Fig. 1 we show the dependence of the structure factor on the block number $p$. Clearly, the dominant length-scale $l \sim 1 / q^{*}$ tends to becomes shorter as the number of blocks increases. This phenomenon is quantified further in Fig. 2 , where $q^{*}$ is shown as a function of number of blocks $p$. This tendency of the dominate length-scale to become shorter as result of increased connectivity in the multiblock systems as compared to the diblock systems has also been observed in small angle x-ray and neutron scattering experiments $[5,6]$. The micro segregation transition occurs formally for the 

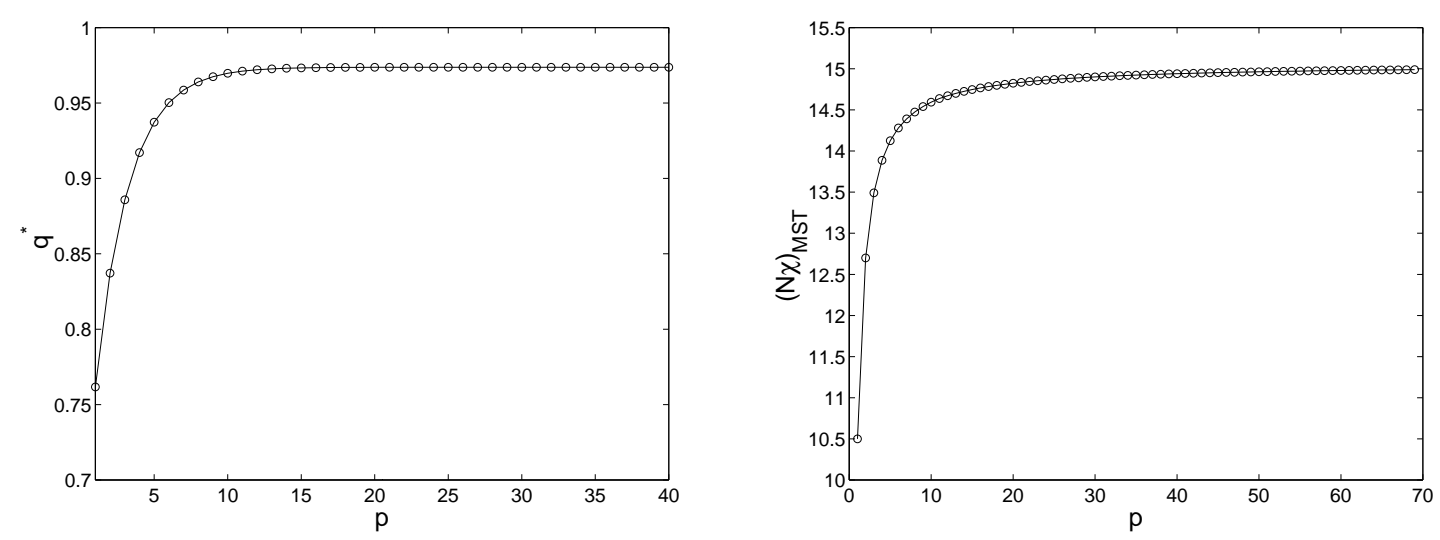

Figure 2: Left: position $q^{*}$ of the structure function peak vs. number of blocks $p$. Right: dependence of the micro segregation transition (MST) on the number of blocks $p$.

temperature $N \chi$ at which the strength of the fluctuations becomes infinite, thus when the peak of the structure factor diverges. It is known that for diblock copolymers this occurs for $N \chi \simeq 10.5$. In the right panel of Fig. 2 we show how the position of the micro segregation transition is affected as more blocks are added to the polymer chains.

\section{Bond Fluctuation Realizations}

Three-dimensional lattice Monte Carlo simulations were performed using the bond fluctuation model[]. This widely used Metropolis-based algorithm enforces excluded volume and non-crossing conditions, has favorable ergodicity properties with physically motivated elementary moves, and allows for implementation of simple interaction potentials between monomers. A cubic, periodic simulation volume of linear dimension $L=48$ cells was used. The density, defined as $\rho=8 N N_{c} / L^{3}$, where $N_{c}$ is the number of monodisperse chains of length $N$, was 0.45 in all cases. Chains were taken to be regular multiblocks with primary structure $\left(A_{m} B_{n}\right)_{p}$, where $A$ and $B$ denote monomer types and $p$ is the number of connected "diblocks" along a given chain; the cases $\phi=m / m+n=0.25$ and 0.5 with $m+n=16$ were considered for $p=2,4,5$, and 6 . A "box potential" repulsive interaction $\epsilon_{A B}=3$ was employed for unlike, non-bonded monomers. Thus, for a given value of $\phi$, the only variables were $N$ and $N_{c}$, with the constraint that $N / N_{c}$ was constant. Each realization was initiated by distributing the individual chains on the lattice with a (biased) random walk, after which the system was equilibrated using athermal conditions (i.e., $\epsilon_{A B}=0$ ) for a sequence of $2 \times 10^{7}$ Monte Carlo steps (MCS), where one MCS is defined as one attempted move, on average, per monomer. The equilibration stage was followed by production run of length $6.4 \times 10^{7}$ MCS; the system configuration was recorded at $1 \times 10^{6}$ MCS intervals for subsequent analysis, which in the present case corresponds primarily to calculation of the collective structure factor for the systems.

The described bond fluctuation method also allows extraction of the structure factor. In Fig. 1 we show data obtained as result of the bond fluctuation simulations for the same parameters as was used to generate Fig. 1. The results are almost in quantitative agreement.

Further, one can depict the exact position of single monomers in the simulations and 

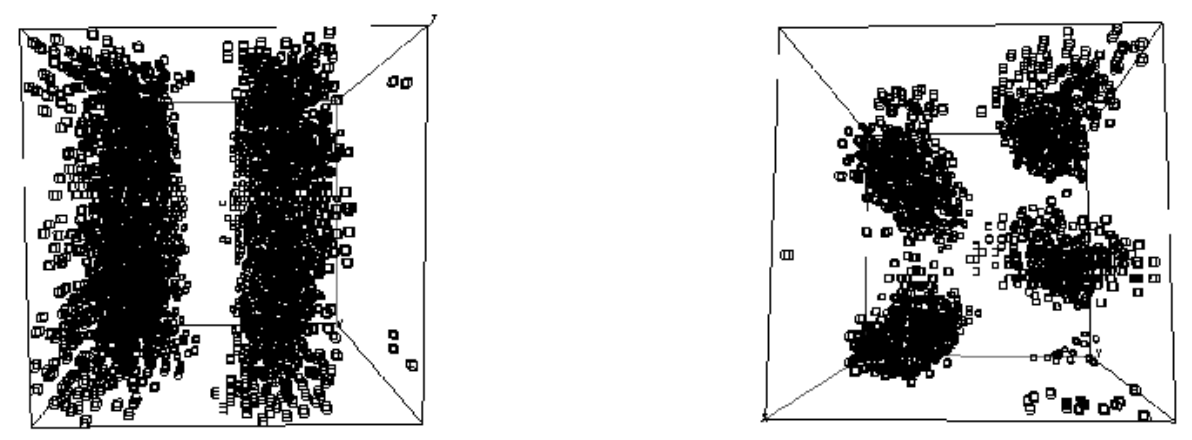

Figure 3: Monomer configuration (only one species shown) for diblock system $(p=1)$, for (left) symmetric blocks $f=0.5$ and (right) asymmetric $(f=0.25)$ polymers.

obtain information of the equilibrium morphology for particular parameter values. Two examples are shown in Fig. 3

\section{Conclusion}

In conclusion we have described how the formalism initially developed by Leibler for diblock copolymers easily can be extended to apply to multiblock copolymer systems. We have described some of the basic results such an extension offers, and demonstrated that the results compare favorably to bond fluctuation simulations and also to small angle scattering experiments. Our future goal is to further extend the theory to apply to more arbitrary architectures of the polymers, and more importantly to extend the theory to describe the fluctuation involved in transitions between ordered phases [7]. Accomplishing this task will potentially give us a unique theoretical tool to understand the elastic and viscoelastic properties of copolymer systems.

\section{Acknowledgments}

We gratefully acknowledge fruitful discussions with S. Chitanvis, E. Kober, and R. Hjelm. Research at Los Alamos National Laboratory is performed under the auspices of the US DOE.

\section{References}

[1] L. Leibler, Macromolecules, 13, 1602 (1980).

[2] T. Ohta, and K. Kawasaki, Macromolecules, 19, 2621 (1986).

[3] M. Doi, and S. F. Edwards, The Theory of Polymer Dynamics, (Clarendon Press, Oxford, 1986). 
[4] P.-G. de Gennes, Scaling Concepts in Polymers Physics, (Cornell University Press, Ithaca, 1953).

[5] S.D. Smith, R.J. Spontak, M.M. Satkowski, A. Ashraf, A.K. Heape, and J.S. Lin, Polymer, 35, 4527 (1994).

[6] R. Hjelm et al., Private communications.

[7] A. Shi, A. Noolandi, R. C. Desai, Macromolecules, 29, 6487 (1996). 